\title{
Morphological and morphometrical assessment of spermathecae of Aedes aegypti females
}

\author{
Tales Vicari Pascini', Marcelo Ramalho-Ortigão², Gustavo Ferreira Martins ${ }^{1 /+}$ \\ ${ }^{1}$ Departamento de Biologia Geral, Universidade Federal de Viçosa, Viçosa, MG, Brasil \\ ${ }^{2}$ Department of Entomology, Kansas State University, Manhattan, KS, USA
}

\begin{abstract}
The vectorial capacity of Aedes aegypti is directly influenced by its high reproductive output. Nevertheless, females are restricted to a single mating event, sufficient to acquire enough sperm to fertilize a lifetime supply of eggs. How Ae. aegypti is able to maintain viable spermatozoa remains a mystery. Male spermatozoa are stored within either of two spermathecae that in Ae. aegypti consist of one large and two smaller organs each. In addition, each organ is divided into reservoir, duct and glandular portions. Many aspects of the morphology of the spermatheca in virgin and inseminated Ae. aegypti were investigated here using a combination of light, confocal, electron and scanning microscopes, as well as histochemistry. The abundance of mitochondria and microvilli in spermathecal gland cells is suggestive of a secretory role and results obtained from periodic acid Schiff assays of cell apexes and lumens indicate that gland cells produce and secrete neutral polysaccharides probably related to maintenance of spermatozoa. These new data contribute to our understanding of gamete maintenance in the spermathecae of Ae. aegypti and to an improved general understanding of mosquito reproductive biology.
\end{abstract}

Key words: mosquito reproductive system - scanning electron microscopy transmission electron microscopy - confocal microscopy - histochemistry

Aedes aegypti is the most important vector of dengue and urban yellow fever viruses, due in some measure to the high reproductive output of Ae. aegypti females. In spite of such high output, Ae. aegypti females are restricted to a single mating event, sufficient to acquire enough sperm to fertilize a lifetime supply of eggs (Rogers et al. 2008). How mosquito females are capable of maintaining live gametes for several days or weeks remains unknown.

Following copulation, insect spermatozoa are housed within the sac-like reservoir formed by the distal and dilated portion of the spermatheca. The reservoir is connected to the bursa copulatrix through spermathecal duct that allows passage of male gametes from the bursa copulatrix to the reservoir following mating or in the opposite direction for egg fertilization. A spermathecal pump, also known as sphincter, is located at the site of attachment of the spermathecal duct to the reservoir. The spermathecal pump of bees and ants is formed by several muscular fibres arranged in different orientations (Martins \& Serrão 2002, Gobin et al. 2006) and functions to prevent gametes from escaping from the reservoir and to control their release during egg fertilization by contracting and relaxing, respectively (Gerber \& Klostermeyer 1970).

Financial support: FAPEMIG, DBG/UFV

TVP is an undergraduate student in the Biology program at UFV and supported by PIBIC/CNPq, GFM is supported by FAPEMIG (CBB-APQ-00394-09) and MRO is supported by NIH (AI074691, AI088051)

+ Corresponding author: gmartins@ufv.br

Received 12 January 2012

Accepted 27 March 2012
The glandular portion of the spermatheca generally consists of clustered cells located just above the reservoir and/or the duct epithelia. Depending on the insect species, the clustered cells have either individual ducts that transport secreted substances directly into the reservoir (Curtin \& Jones 1961) or a common glandular duct (Lawson \& Thomas 1970, Martins et al. 2008). Secreted substances are thought to include nutrients that keep the spermatozoa viable during the entire reproductive life of the adult female (Schoeters \& Billen 2000, Klenk et al. 2004).

In Apis mellifera, the spermatheca participates in the maturation and viability of the spermatozoa, creating a suitable environment by isolating gametes from the haemolymph and by synthesizing antioxidants (e.g. catalase, glutathione- $S$-transferase and superoxide dismutase) that protect gametes against reactive oxygen species (Collins et al. 2004, Kumano et al. 2009, den Boer et al. 2009). In addition, genes expressed in the spermatheca of Drosophila simulans are involved in controlling the viscosity of the internal milieu of the spermathecal lumen, as well as in sugar metabolism to provide energy sources to the gametes and in antifungal defence (Prokupek et al. 2008).

The ultrastructure of the Ae. aegypti spermatheca was described previously (Christophers 1960, Clements \& Potter 1967). However, morphological studies combining histology, histochemistry, confocal and scanning and transmission electron microscopes have never been done. Here, we combined histology, histochemistry and microscopy approaches to reveal general and detailed aspects of Ae. aegypti spermathecae morphology, including cell composition and nuclear size. The morphology of the spermathecae, as well as the morphometry and the number of gland cells and their cytoskeleton, do not differ between unmated to mated Ae. aegypti females, showing that just the organ filling by male gametes is 
not a prerequisite to change organ morphology. However, these results indicate that gland cells produce and secrete polysaccharides (probably related to the maintenance of spermatozoa) even before mating. These data bring new insights into understanding how gametes are maintained in Ae. aegypti and improve the general body of knowledge about the reproductive biology of mosquitoes.

\section{MATERIALS AND METHODS}

Mosquitoes - Ae. aegypti strain PP-Campos (Campos dos Goytacazes, state of Rio de Janeiro, Brazil) was obtained from a colony maintained at the Laboratory of Insect Pheromones (Institute of Biotechnology Applied to Agricultural Sciences, Federal University of Viçosa, state of Minas Gerais, Brazil). Two groups of pupae were separated into two different cages: one cage with females and another with males and females (2:1 ratio). Three days after emergence, males were removed from the cage. Adult mosquitoes were kept at $25-28^{\circ} \mathrm{C}$ with $70-80 \%$ relative humidity and in a 12:12 light-dark cycle. Adults also were maintained on $50 \%$ honey solution ad libitum. Non-inseminated (i.e., sperm-less) females collected from cages containing males were discarded during our analyses.

Dissection and sample preparation - Ten to 13-day-old females (virgin and inseminated) were dissected separately under a stereomicroscope in $0.1 \mathrm{M}$ sodium phosphate buffer (PBS), pH 7.2. The last two abdominal segments were removed with the spermathecae attached. Samples were transferred to a fixative solution containing $2.5 \%$ glutaraldehyde, $0.1 \mathrm{M}$ cacodylate buffer, $\mathrm{pH}$ 7.2. After dissection, samples were maintained in the fixative solution until further use in the experiments described below.

Light microscopy - Spermathecae from 30 virgin and 30 inseminated females were dehydrated in a crescent series (30-100\%) of ethanol, followed by clarification in xylene for whole mount preparations. For histology and histochemistry, fixed samples were rinsed in PBS, dehydrated in a crescent series of ethanol (70-100\%) and embedded in Historesin (Leica). Thin sections $(4 \mu \mathrm{m})$ were stained with haematoxylin and eosin. Approximately 20 whole spermathecae from virgin and 20 spermathecae from inseminated females were washed in PBS, incubated $16 \mathrm{~h}$ in $0.5 \%$ glycine and subjected to the periodic acid Schiff (PAS) reaction for detection of neutral polysaccharides. Samples were dehydrated, embedded and sectioned as described earlier. In addition, 10 fixed spermathecae from each of the of two female groups were washed three times with PBS, stained with 4',6-diamidino-2-phenylindole, $0.5 \mathrm{~g} / \mathrm{mL}$ solution (DAPI) (Sigma-Aldrich) at room temperature for $1 \mathrm{~h}$ and protected against light, mounted in sucrose and observed using an Olympus BX60 fluorescence microscope using a WU filter. Seriated pictures were taken under the same microscope coupled with an Olympus Q-colour3 digital camera.

Spermathecae were fixed in 4\% formaldehyde solution in PBS, pH 7.2 for a period of $30 \mathrm{~min}$ and washed three times in PBS for 5 min each. Samples were then incubated for $30 \mathrm{~min}$ in fluorescent Phalloidin-FITC (Sigma-Aldrich) diluted $1: 100$ in $0.1 \%$ Triton X-100 and washed as before. Prior to analysis, samples were mounted with the anti-fading solution Mowiol (Longin et al. 1993 and reference therein). Images were obtained using multiple confocal sections from a Zeiss laser confocal microscope 5100 .

Morphometry - Gland cell nuclei attached to the spermathecal reservoir were counted and measured (diameter) according to their distribution throughout the large and small spermathecae of virgin and inseminated females. For each group, 10 females were used and the nuclei arbitrarily selected from DAPI stained whole mounts. Nuclear number and sizes (diameter) were determined with the software Image ProPlus ${ }^{\mathrm{TM}}$ and compared using an independent two-sample t-test.

Scanning electron microscopy (SEM) - Glutaraldehyde-fixed samples were post-fixed in $1 \%$ osmium tetroxide in $0.1 \mathrm{M}$ sodium cacodylate buffer $\mathrm{pH}$ 7.2. Samples were dehydrated in a crescent series (30-100\%) of acetone, critical-point dried using $\mathrm{CO}_{2}$ and sputter coated with gold for observation under SEM (LEO 1430VP). Spermathecae from 10 virgin and 10 inseminated females were gently opened with the aid of very thin entomological needles before metallization, in order to observe their interior and content.

Transmission electron microscopy (TEM) - Spermathecae were transferred to $2.5 \%$ glutaraldehyde in $0.1 \mathrm{M}$ sodium cacodylate buffer $\mathrm{pH} 7.2$ and post-fixed in $1 \%$ osmium tetroxide in the same buffer. Samples were dehydrated in a crescent series acetone and embedded in Spurr's resin (Electron Microscopy Sciences, EMS, Hatfield, PA). Semi-thin sections were stained with 1\% toluidine blue-borax and ultra-thin sections were stained with uranyl acetate and lead citrate. After the staining, ultra-thin sections were washed in distilled water and analyzed under a Zeiss EM109.

\section{RESULTS}

Light microscopy - Ae. aegypti female has three spermathecae, one large and two small, that are visible under the light microscope in both virgin and in inseminated females. Each spermathecae is composed of a round and dark-brown reservoir connected to the bursa copulatrix by translucent spermathecal duct. In virgin females, the walls in the spermathecal reservoirs have deep invaginations not easily observed in inseminated females (Fig. 1A). Two-three days after insemination, the spermatozoa are organized in a circular fashion inside the reservoir lumens of large and small spermathecae and also underneath the spermathecal reservoir cuticle (Fig. 1B).

Histological sections indicate that spermathecal reservoirs are lined by a thick cuticle, which is continuous with the cuticle of the spermathecal duct. However, the cuticle is narrower at the point of attachment of the spermathecal duct with the reservoir (Figs 1C, 2A). External to the reservoir cuticle, a single, very thin epithelium with cells displaying a flattened nucleus is visible (Fig. 2C). Positioned at the transition between the reservoir and the duct are the spermathecal glands made of "swollen cells" with well-developed nuclei. These cells are connected to the reservoir lumen through individual 
ductules lined by a thin cuticle that also is continuous with the reservoir cuticle (Figs 1C, 2A, E). Spermathecal ducts are also lined by a thick cuticle covered externally by a single columnar epithelium (Fig. 2A, D). External to the duct epithelium are glandular cells (Fig. 2D) that morphologically resemble those observed in the spermathecal gland. In addition to gland cells, muscular fibres forming the spermathecal pump or sphincter are seen near duct-reservoir junction (Fig. 1C).

In virgin females, the reservoir lumens contain no gametes. In contrast, spermatozoa are densely packed inside the lumens of the inseminated females and appear as parallel bundles when their heads are cross-sectioned, as seen in Fig. 2B. The luminal content of reservoirs in virgin and inseminated females is positively stained for the PAS reaction. Gland cells appear as PAS-positives, while the reservoir epithelia are negatives (not shown); the content within the gland cell ductule is strongly stained for PAS reaction (Fig. 2E).

Under fluorescence microscopy, DAPI staining revealed that the nuclei of the epithelial cells of the reservoir are not homogenously distributed (Fig. 3A). Also, gland cell ductules of spermathecal glands appear enlarged in comparison to gland cells of the spermathecal duct (Fig. 3B). In whole mounts we also observed that the spermathecal duct and glandular cell cuticles are light blue-fluorescent (Fig. 3B, C) and that the ducts of two small spermathecae fuse shortly before the bursa copulatrix, where duct gland cells are absent (Fig. 3C). Confocal images revealed that the cytoskeleton was distinctly fluorescent in the apex of glandular cells (Fig. 3D, E).
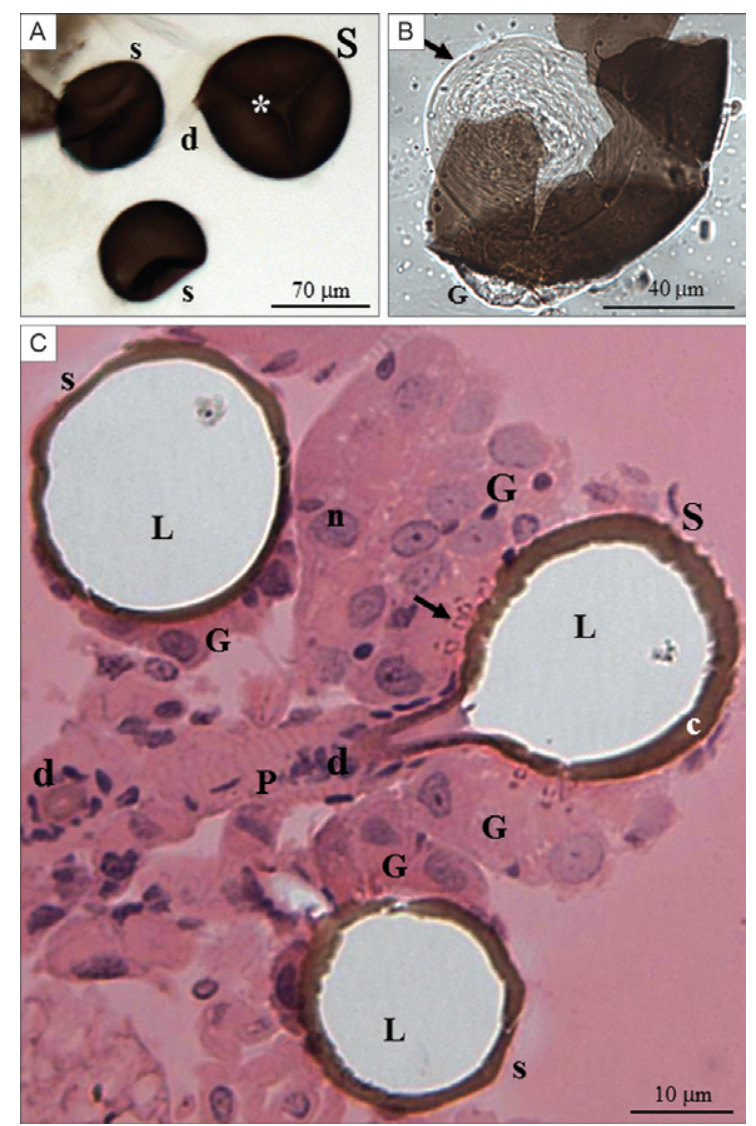

Fig. 1: general view of the three Aedes aegypti female spermathecae. A: whole mount depicting the one large (S) and the two small (s) spermathecae reservoirs from a virgin Ae. aegypti female with dark-brown reservoirs. All three reservoirs are empty with the presence of infolds (asterisk) on their wall. The transparent large spermathecal duct is indicated (d); B: whole mount showing a disrupted small spermatheca from an inseminated female filled with spermatozoa (arrow) in a circular array. The spermathecal gland is marked (G); C: histological section showing the reservoirs of the large (S) and the two small (s) spermathecae from an inseminated female. The reservoir lumen (L) is lined by a thick cuticle (c) that is continuous with the duct cuticle (d). The spermathecal gland are positioned externally to the reservoir and the pump (P) is formed by muscular tissue located above the duct epithelium. The arrow points to the reservoir-duct transition; n: cell gland nucleus.
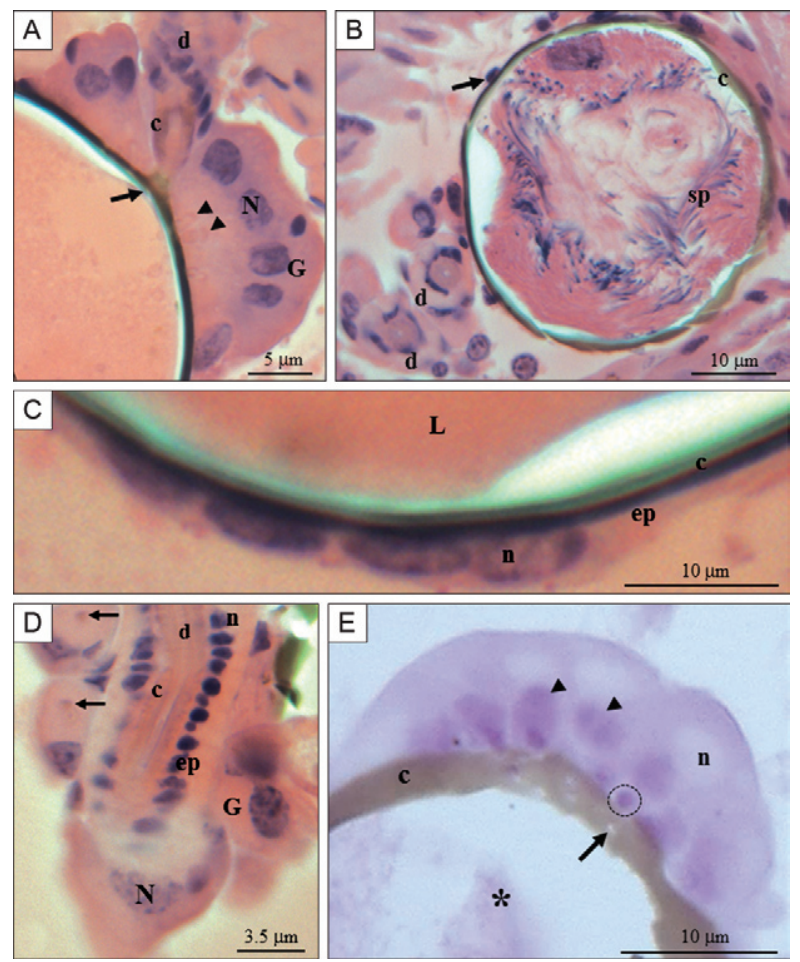

Fig. 2: histological sections showing Aedes aegypti female spermathecae stained with haematoxylin-eosin (A-D) and periodic acid Schiff (PAS) reaction (E). A: section from one of the small spermathecae from a virgin female with details of the spermathecal gland $(\mathrm{G})$ formed by cells displaying well-developed nucleus $(\mathrm{N})$ and connected to the reservoir lumen by cell ductules (arrowheads). The arrow points to the opening of the spermathecal duct (d). The duct cuticle (c) is continuous with the reservoir cuticle; B: section of the small spermatheca of an inseminated female showing the spermatozoa (sp) surrounded by the cuticle (c). Arrow points to the nucleus of the flattened epithelial cell found on the reservoir wall; C: reservoir wall of a small spermatheca from a virgin female formed by flattened epithelial cells (ep) (L: reservoir lumen; $\mathrm{n}$ : cell nucleus); D: longitudinal section of a duct of a large spermatheca of an inseminated female displaying a columnar epithelial cells with spermathecal gland positioned externally (arrows: gland cell ductules); E: PAS-stained section showing PAS-positive regions around the cell ductules (arrowheads) of a spermatheca from a virgin female. Note the presence of gland cells ducts filled with material strongly stained by PAS (dotted circle) and PAS-positive material in the reservoir lumen (*). Arrow points to the cell ductule openings in the cuticle. 
In addition, the epithelial cells also showed a cytoplasm negative for the Phalloidin-FITC fluorescent stain.

The number of cells present in the spermathecal gland does not differ significantly between virgin and inseminated females. A total of $70.70 \pm 6.79$ (mean \pm standard deviation) cells were observed in the large spermatheca of virgin vs. $79.80 \pm 6.04$ cells in the large spermatheca of inseminated Ae. aegypti females. For the two smaller spermathecae, $33.50 \pm 7.25$ cells were detected in virgins vs. $37.65 \pm 6.51$ in inseminated females. Considering both large and small spermathecae, the mean size of nuclear spermathecal gland cell diameter is $3.44 \pm 1.14$ for virgin vs. $3.35 \pm 1.15 \mu \mathrm{m}$ for inseminated females and they also do not differ significantly.

SEM - Under the SEM, spermathecae of virgin and inseminated Ae. aegypti females have round reservoirs with attached spermathecal glands located at the reservoir-duct junction. Spermathecal ducts are tubular structures attached to reservoir and with individual glandular cells attached along their surface (Fig. 4A). At the reservoir external surface, the nuclei of epithelial cells are only seen when the basal lamina is occasionally broken (Fig. 4A, inset).
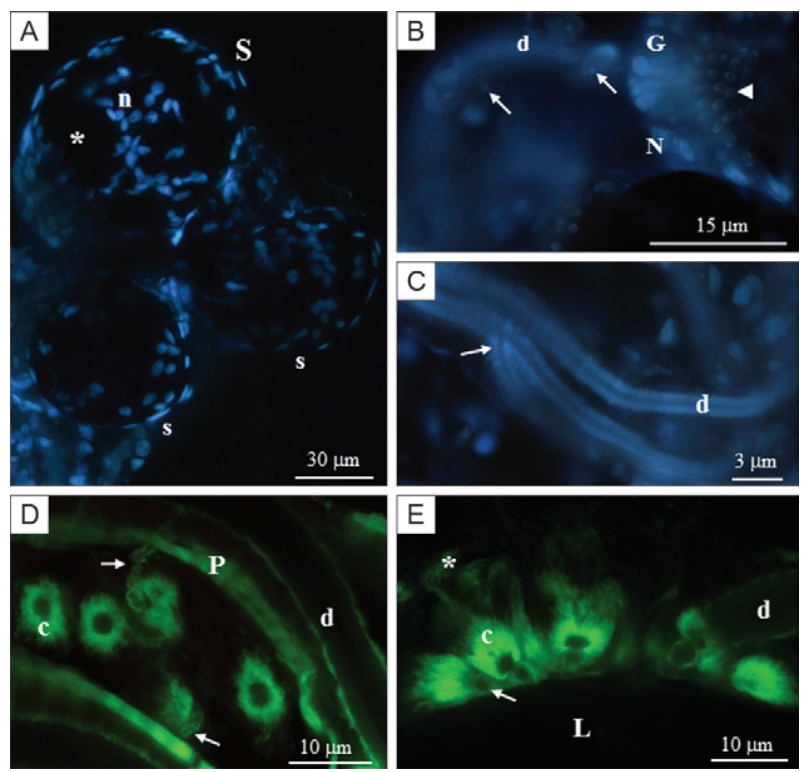

Fig. 3: whole mounts of the spermathecae of virgin Aedes aegypti females stained with 4',6-diamidino-2-phenylindole, $0.5 \mathrm{~g} / \mathrm{mL}$ solution (DAPI) (A, B, C) and Phalloidin-FITC (D, E). A: three spermathecal reservoirs are depicted in the figure, the large (S) and the two small (s). Asterisk indicates area without cell nuclei and where nuclei (n) appear concentrated; B: view of the large spermatheca showing the spermathecal gland (G). Note the fluorescent blue spermathecal gland cell nuclei (N) and the gland cell ductules (arrowhead). Also, gland cells with their ductules (arrows) are attached along spermathecal duct (d); C: duct with fluorescent cuticle. Arrow points to the junction between the ducts of two small spermathecae; D, E: laser confocal microscope analyses of glandular cells attached to the spermathecal duct (D) and reservoir (E) with the cytoskeleton (actin filaments) distinctly labelled by the greenfluorescent stain. Note that labelling is strong at cell apex (c) close to the cell ductule (arrows) and it is weak at the cell base (asterisk); (L: reservoir lumen; P: spermathecal pump).
Each reservoir's wall cuticle is smooth except in the region just beneath the spermathecal glands where protuberances corresponding to openings of the gland cell ductules through the reservoir cuticle are seen (Fig. 4B). The content of the spermathecal reservoir in inseminated Ae. aegypti females is composed of densely packaged "wool ball" bundles of spermatozoa stored just underneath the reservoir cuticle in either small and large spermathecae (Fig. 5A, B).

TEM - Under the TEM, the reservoir epithelium is very thin with the cells tightly attached to each other. Flattened nuclei and cytoplasms poor in organelles are also seen. A thick, electron-dense cuticle separates epithelial cells from reservoir lumen (Fig. 6A).

In contrast to reservoir epithelial cells, spermathecal gland cells in either groups of females are swollen and with well-developed nuclei. Their cytoplasm apex is rich in mitochondria where the cell membrane has convolutions representing microvilli that extend towards the gland cell ductile (Fig. 6B, C). The lumen of the ductule is filled with electron-dense material (Fig. 6B). Flattened epithelium cells are seen between gland cells and reservoir cuticle (Fig. 6C).

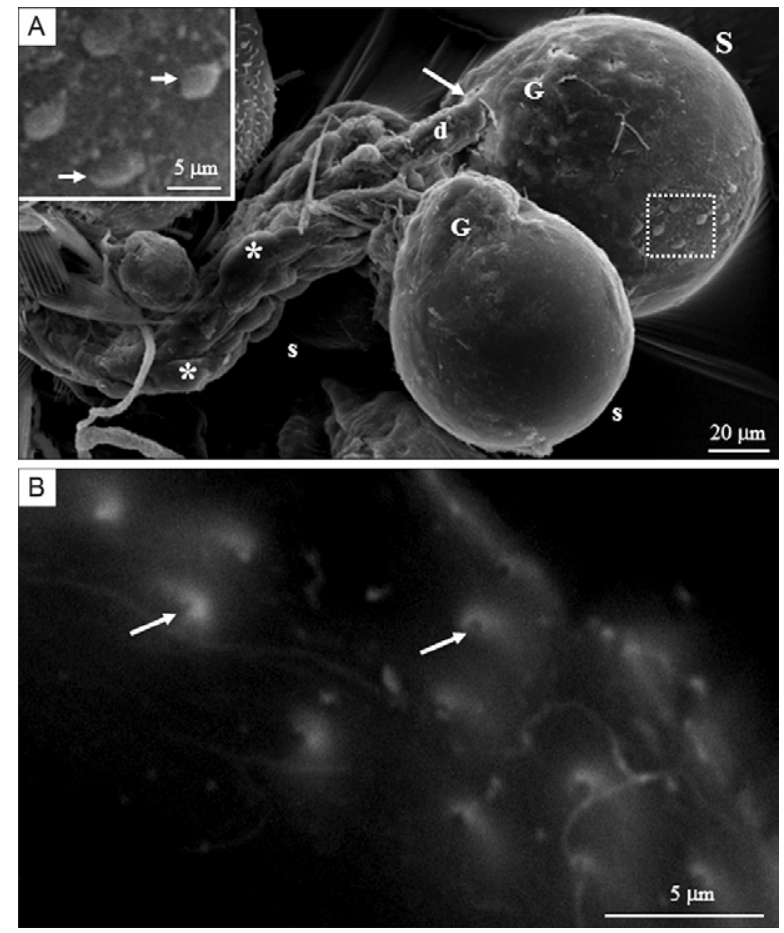

Fig. 4: scanning electron microscopy (SEM) showing of the spermathecae of inseminated Aedes aegypti females. A: general SEM view of inseminated Ae. aegypti depicting the three spermathecal reservoirs [two small (s) and one large (S)]. Spermathecal glands (G) are located above the reservoirs. The large arrow points to the reservoir-duct transition. The duct (d) is cylindrical with the glandular cells $(*)$ surrounding it. The inset shows a detail of the reservoir wall surface (dotted line square) with the protuberances that correspond to nuclei from epithelium cells (small arrows); B: view of the reservoir cuticle with a smooth internal surface, except in the region adjacent to the spermathecal gland, where several gland cell ductule openings are visible (arrows). 
Detailed views of spermatheca ducts (Figs 7, 8) display duct lumens lined by a very thick electron-dense cuticle surrounded by the duct epithelium. This epithelium is formed by cells displaying a cytoplasm-filling nucleus that almost entirely covers the surface of the cytoplasm. External to this epithelium are the glandular cells and the muscular fibres corresponding to the spermathecal pump. The glandular cells resemble the cells from the spermathecal gland with respect to the presence of microvilli and a large number of mitochondria on their apex. Here, the cell ductules are thinner and longer than those from the spermathecal gland cells (Fig. 7). Spermatozoa tails (with axonema and mitochondrial derivatives) were serendipitously observed in transversal sections of ducts of inseminated Ae. aegypti females, specifically near the attachment point in the spermathecal reservoir (Fig. 8A). As the luminal content is lost during sectioning of the
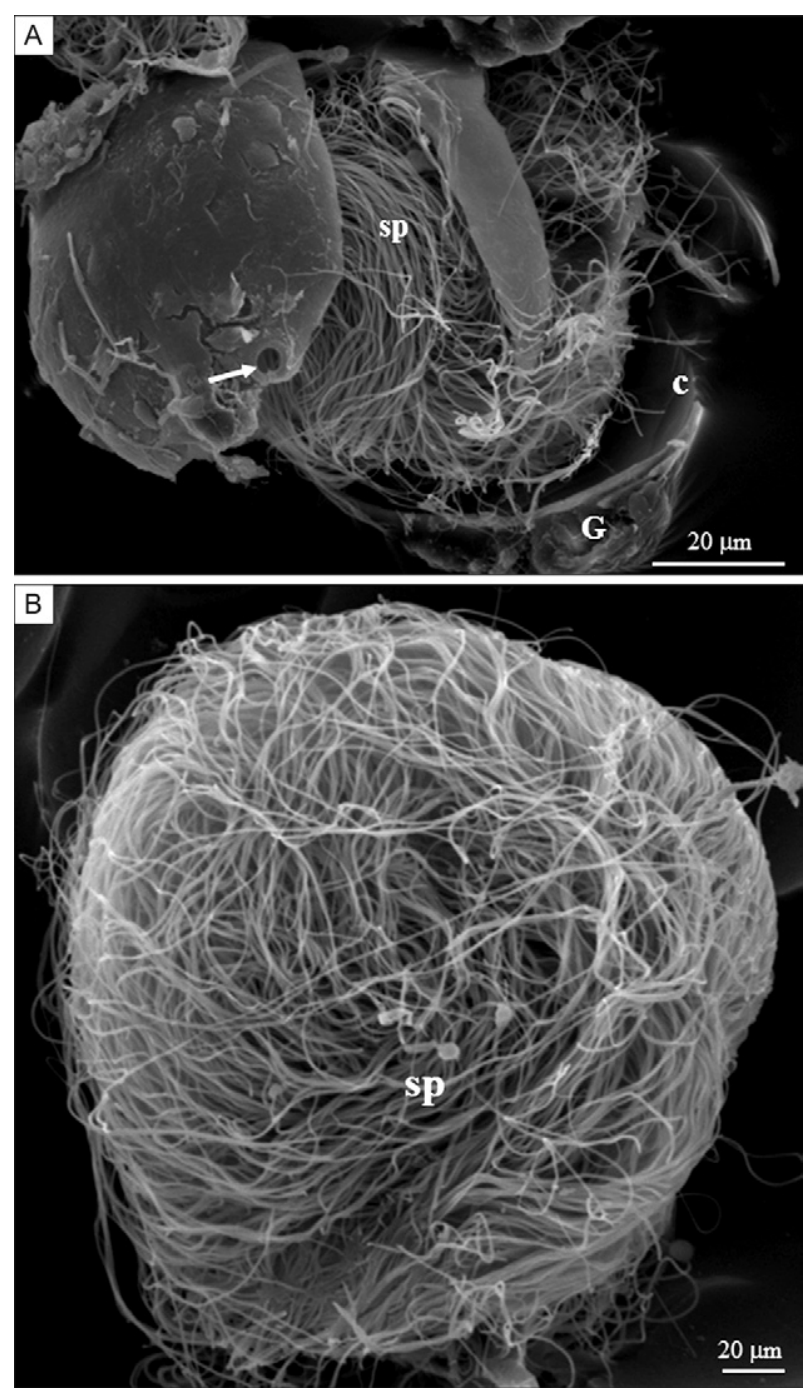

Fig. 5: scanning electron microscopy of the small (A) and large (B) spermathecae from inseminated Aedes aegypti females. Spermathecal reservoirs were disrupted using an entomological needle, exposing the densely packed spermatozoa (sp). Arrow points to the attachment of spermathecal duct in the reservoir. Note the parallel array displayed by the spermatozoa. c: cuticle; G: spermathecal gland. reservoirs, this was not frequently observed. Finally, the epithelial cells of the spermathecal duct display convoluted cell membranes facing the duct cuticle and show electron-dense infoldings associated with such irregular outer surfaces (Fig. 8B).

\section{DISCUSSION}

In spite of its key role for the mosquito reproductive success, few studies have focused on structural features of mosquito spermatheca. Here, we assessed details of the morphology and morphometry of the spermatheca of the major disease vector, Ae. aegypti, before and after copulation. The Ae. aegypti spermatheca is formed by three individual reservoirs (or spermathecae), a large (100 $\mu \mathrm{m}$ in diameter) and two smaller (75 $\mu \mathrm{m}$ in diameter). This is similar to what is seen in Ae. albopictus (Barreto et al. 2008) and Culex sp. (GF Martins, unpublished observations) and distinct from Anopheles gambiae, which display a single spermatheca (Klowden \& Chambers 2004). Also, likely in these three mosquito species, in Ae. aegypti each reservoir is nearly perfectly round with a
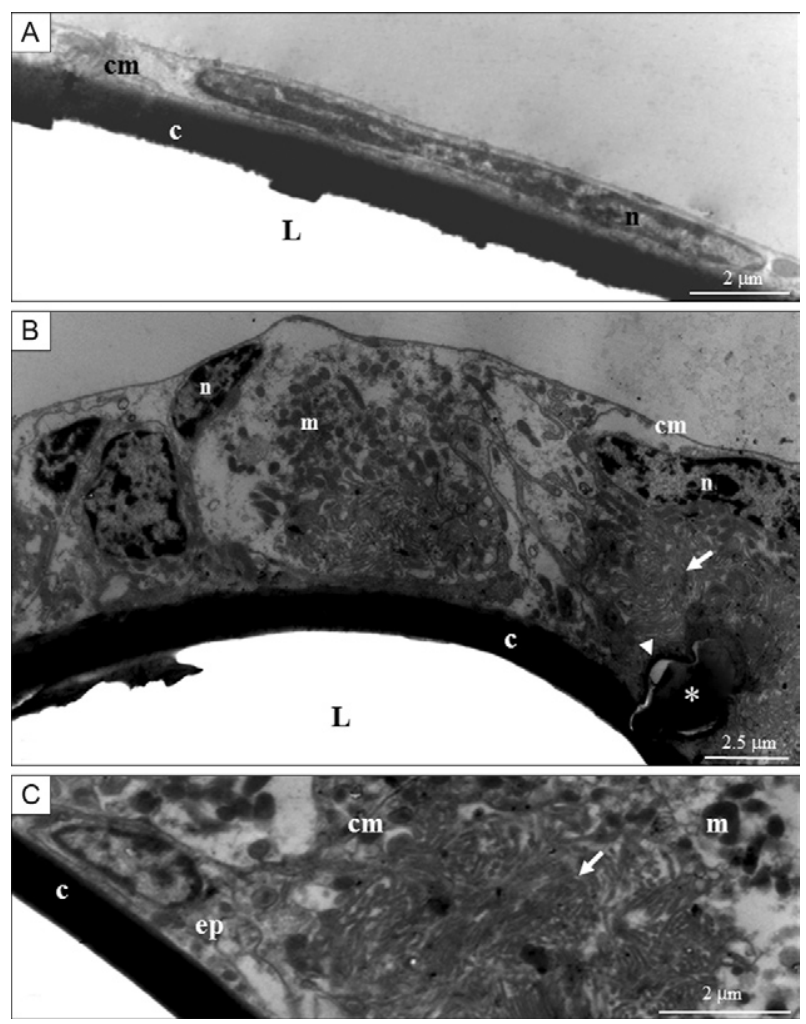

Fig. 6: transmission electron microscopy of the reservoir epithelium and spermathecal gland from virgin Aedes aegypti female. A: flattened epithelial cell and nucleus (n) in close association with the reservoir cuticle (c). The cytoplasm in these cells is poor in organelles. The content in the lumen of the spermathecal reservoir (L) was lost during ultramicrotomy (cm: cell membrane); B: gland cells with welldeveloped nuclei (n), and a cytoplasm rich in mitochondria (m). Cell ductule $(*)$ is depicted lined by a thin cuticle (arrowhead) continuous with the cuticle (c). Ductules are filled by periodic acid Schiff-positive electron-dense homogeneous material (Fig. 2E). Arrow points to microvilli found on cell apex; C: epithelial cell (ep) located underneath gland cells and immediately above the cuticle; arrow: cell microvilli. 
dark-brown colour due to the thick cuticle lining of their lumens. The spermathecal ducts and gland cell ductule cuticles are fluorescent under ultraviolet light and are also known to contain resilin, an elastic protein found in insect cuticle that confers the spermathecal duct its ability to stretch (Curtin \& Jones 1961, Clements \& Potter 1967). Microscopic inspection of whole mounts revealed that the spermatozoa can be observed inside either the large or the small spermathecal reservoirs, despite the thickness of the cuticle. The gametes are packaged in parallel arrays resembling a bundle of long and tiny cells, as determined via analyses performed in whole organs or via the histological sections and the SEM.

Curiously, in Ae. aegypti, the length of most spermatozoa varies from 250-300 $\mu \mathrm{m}$ (Klowden \& Chambers 2004) and they must fit within the large and small spermatheca reservoirs (100 and $75 \mu \mathrm{m}$ in diameter, respectively). So, as gametes are much bigger than the diameters of the luminal reservoirs in which they are stored, the circular array configuration allows their accommodation and organization at high densities (up to 600 spermatozoa/spermatheca) (Jones \& Wheeler 1965). According to previous observations, such packaging of sperm at high density maximizes the number of cells that can be stored in an organized manner, whereas the circular cell movement of live spermatozoa seems to be a pre-requisite for the release of single spermatozoa from the insect spermathecae (Werner et al. 1999, Al-Lawati et al. 2009).

Our data indicate that despite their size differences, the three spermathecae have the same general morphology and cell-type composition in virgin and inseminated $A e$. aegypti females. One exception, however, is the spermathecal gland-cell number, which in the large spermath-

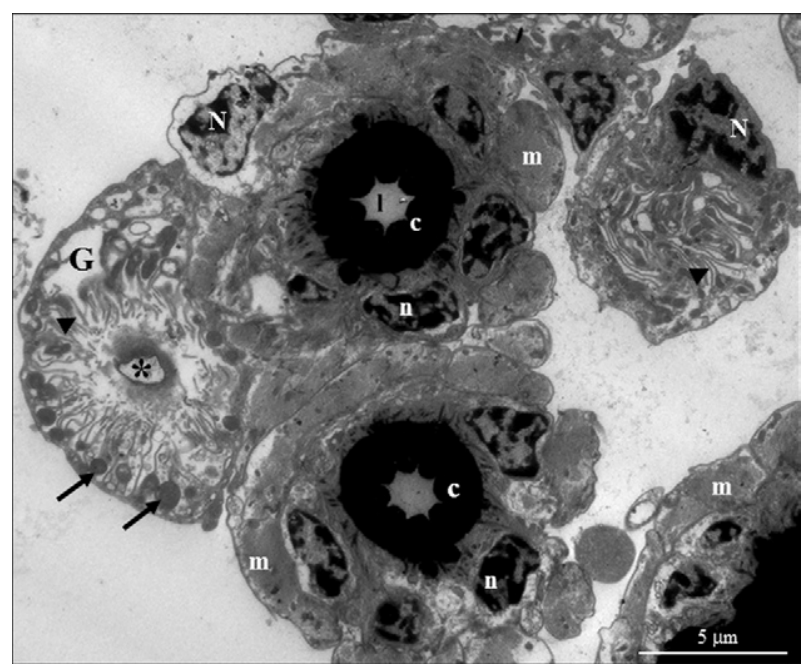

Fig. 7: transmission electron microscopy of transversally sectioned ducts of the small spermathecae of a virgin Aedes aegypti female. Ducts are lined by thick and electron-dense cuticle (c) and surrounded by tightened cells with nuclei (n) rich in condensed chromatin. Externally to the duct epithelia, spermathecal muscular fibres from the spermathecal pump (m) and duct gland cells (G) are found. Gland cells display well-developed nuclei $(\mathrm{N})$ and microvilli (arrowhead) associated with mitochondria (arrows). Asterisk indicates the gland cell ductule. 1: duct lumen. ecae is roughly twice that of the small ones. The large number of glandular cells present in the large spermatheca might be associated with its need to provide nourishment to more gametes in comparison to the smaller spermathecae. Under light microscopy, epithelial and glandular cell types can be distinguished in the spermathecae. As observed in spermathecae from other insects, these cells are quite different from each other in terms of morphology and ultrastructure and such differences may be associated with their different roles (Tombes \& Roppel 1972, Lay et al. 1999, Staccomi \& Romani 2010).

In most insects, the epithelial cells of the reservoir are columnar with abundant organelles (mainly mitochondria) and with cell membrane in-foldings in their apex and base (Dallai 1975, Gobin et al. 2006, Martins et al. 2008). In Ae. aegypti, these epithelial cells form very thin $(0.1-0.4 \mu \mathrm{m})$ layers, except where their nuclei expand, causing these layers to increase to up to $3 \mu \mathrm{m}$ (Clements \& Potter 1967). The primary function of these cells is to secrete and maintain the spermathecal cuticle and the subcellular characteristics suggest a role in
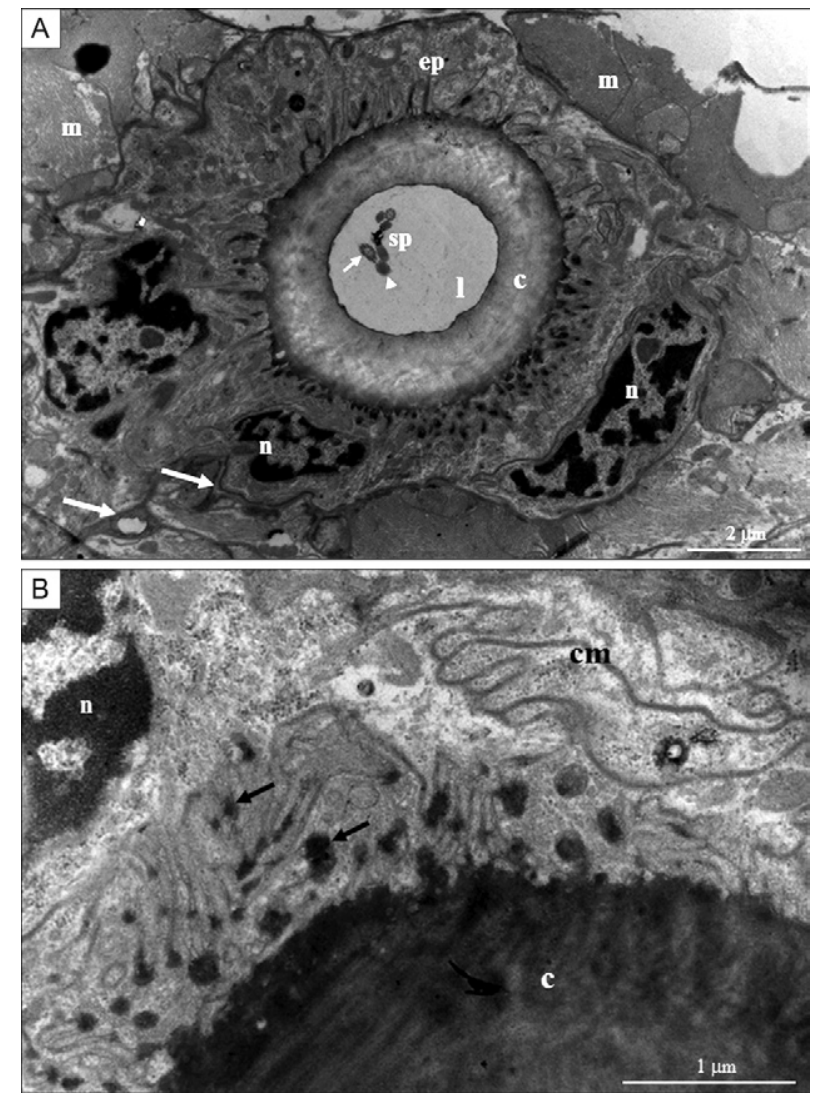

Fig. 8: transmission electron microscopy showing details of the epithelium in the transition zone between spermathecal duct and reservoir of an inseminated Aedes aegypti female. A: epithelial cells (ep) externally surrounded by muscular fibres (m) of the spermathecal pump. Note two sectioned spermatozoa tails (sp) inside lumen (1) (arrowhead: mitochondrial derivative; big arrows: cell ductile; c: cuticle; $\mathrm{n}$ : epithelium cell nuclei; small arrow: axonema); B: cytoplasm from epithelial cell displaying convoluted membranes $(\mathrm{cm})$ in contact with the cuticle. Electron dense materials (arrows), likely part of the cuticle, are observed between infolds of the membrane. 
transport of substances from haemolymph to spermathecal lumen. Dallai (1975), Gobin et al. (2006) and Martins et al. (2008) suggested that the spermathecal epithelium provides a suitable osmotic environment for spermatozoa by transporting substances (e.g. electrolytes) from the haemolymph, while isolating the reservoir lumen from the body cavity. However, results of our TEM analysis seem to indicate that the epithelial cells in $A e$. aegypti are not involved with this transport. The epithelial cells have few organelles, such as mitochondria, and do not have cell membrane specializations, such as in-foldings, and are tightly attached to each other. Further, our results suggest that Ae. aegypti spermathecal epithelial cells are restricted to secrete the cuticle, to house and isolate the sperm from the haemolymph (in conjunction with thick cuticle), and do not participate in active transport of substances as suggested for other insects (Dallai 1975, Gobin et al. 2006, Martins et al. 2008).

Adult mosquitoes like Ae. aegypti have a short lifespan in comparison to ants and queen bees, which live for several months or years, store millions of gametes to fertilize thousands or millions of eggs and must store gametes during a long reproductive life (Al-Lawati et al. 2009). In light of their relatively short life (Muir \& Kay 1998, Tejerina et al. 2009), mosquitoes have no demand for such large spermathecae with well-developed columnar epithelium. Also, the number of eggs laid by mosquitoes is relatively less than in other insects and specifically for Ae. aegypti (Strickman 2006).

Ae. aegypti spermatheca has a single gland formed by cells clustered in the attachment of spermathecal ducts and reservoir walls (Curtin \& Jones 1961). These morphological features differ from those of bees (Schoeters \& Billen 2000, Martins \& Serrão 2002, Souza et al. 2007), ants (Wheeler \& Krutzsch 1994, Gobin et al. 2006) and paper wasps (Martins et al. 2005); each of which have a pair of well-developed spermathecal glands attached to the reservoir. In addition, Ae. aegypti glandular cells are individually and externally attached to spermathecal ducts (Curtin $\&$ Jones 1961). The two types of glandular cells found in Ae. aegypti spermathecae share microvilli at their apex and have large numbers of mitochondria. They also stain strongly with Phalloidin-FITC, suggesting that at this location of the cell the cytoskeleton is involved in a structural role and in assisting in cell transport. Similar results were reported in the spermathecal gland cells of Anastrepha suspensa (Diptera: Tephritidae) (Fritz \& Turner 2002) and such subcellular characteristics are compatible with their predicted secretory role (Staccomi \& Romani 2010). The PAS-reaction confirmed the presence of neutral polysaccharides in the gland's cell apex, ductules and reservoir lumen, even in virgin Ae. aegypti females, in support of their role as secretory cells. Sugars present in the spermathecal lumen likely provide energy for spermatozoa during their storage inside the reservoir and their transport through the spermathecal duct. Despite the absence of sperm in Ae. aegypti females $24 \mathrm{~h}$ after emergence, their spermathecal content is positive for sugars as previously reported for the sand fly Phlebotomus papatasi (Ilango 2005). Likely, sugars are secreted not only in the presence of gametes, but also to prepare the organ to receive male gametes as reported in the beetle Sitophilus granarius (Bhatnagar \& Musgrave 1970) and in several bumblebee species (Schoeters \& Billen 2000).

Mosquito females typically mate only once and males can inseminate several females (Foster \& Walker 2009). During copulation, the male deposits a mixture of sperm and accessory gland secretion in the female's bursa copulatrix, causing the female to become unreceptive to other males. Male seminal fluid proteins have been shown to influence female reproductive and feeding behaviours in many insects (reviewed in Gillot 2003, Avila et al. 2010). In addition, relative to virgin females, inseminated females have increased egg development and oviposition rates. These changes in inseminated females appear to be induced by molecules produced in male accessory glands and transferred to the female during mating as suggested by Avila et al. (2010) and recently confirmed for $A n$. gambiae (Thailayil et al. 2011). A catalogue containing 145 male-derived proteins was recently identified, some of which potentially associated with post-insemination behaviour effects (Sirot et al. 2011). In addition to affecting female behaviour, the spermathecal fluid also participates on the maturation of the spermatozoa after mating (Giuffrida et al. 1995). Besides spermatozoa, mosquito spermathecae also store proteins from male seminal fluid (Sirot et al. 2008, 2011, Thailayil et al. 2011). We speculate that in spite of the presence of a relatively thick spermathecal cuticle $(0.7 \mu \mathrm{m})$ (Clements \& Potter 1967) male proteins might be capable of reaching the haemolymph. Thus, the spermathecae in Ae. aegypti may play an important role in the insect's behaviour.

In this paper we provide a detailed description and comparative morphological analysis of the spermathecae of virgin and inseminated Ae. aegypti females by combining different microscopy analyses. Our results indicate that the morphology and morphometry of Ae. aegypti spermathecae do not change from unmated to mated females and the number of gland cells also remains unchanged. Nevertheless, the abundance of mitochondria, actin filaments and microvillae in the mosquito spermathecal gland cell apex is suggestive of secretory activities and our data show that gland cells produce and secrete polysaccharides, probably related to the maintenance of spermatozoa, even before mating. These findings are relevant for our understanding of the reproductive biology of mosquitoes and may contribute to novel strategies for vector control.

\section{ACKNOWLEDGEMENTS}

To Dr Paulo FP Pimenta (Fiocruz/MG), for providing the mosquito eggs used to establish the Ae. aegypti colony at UFV, and Centre for Microscopy and Microanalysis (UFV), for technical assistance.

\section{REFERENCES}

Al-Lawati H, Kamp G, Bienefeld K 2009. Characteristics of the spermathecal contents of old and young honeybee queens. $J$ Insect Physiol 55: 117-122.

Avila F, Sirot LK, LaFlamme BA, Rubinstein CD, Wolfner MF 2010. Insect seminal fluid proteins: identification and function. Annu Rev Entomol 56: 21-40. 
Barreto M, Burbano ME, Barreto P 2008. Gynandromorphs in mosquitoes (Diptera: Culicidae) from Valle del Cauca, Colombia. $\mathrm{Ne}$ otrop Entomol 37: 296-300.

Bhatnagar RDS, Musgrave AJ 1970. Aspects of histophysiology of the spermathecal gland of Sitophilus granarius (L.) (Coleoptera). Can J Zool 49: 275-277.

Christophers R 1960. Aedes aegypti (L.), the yellow fever mosquito. Its life history, bionomics and structure, Cambridge Univ Press, New York, 314 pp.

Clements AN, Potter SA 1967. The fine structure of the spermathecae and their ducts in the mosquito Aedes aegypti. J Insect Physiol 13: $1825-1836$.

Collins AM, Williams V, Evans JD 2004. Sperm storage and antioxidative enzyme expression in the honey bee, Apis mellifera. Insect Mol Biol 13: 141-146.

Curtin TJ, Jones JC 1961. The mechanism of ovulation and oviposition in Aedes aegypti. Ann Entomol Soc Am 54: 298-313.

Dallai R 1975. Fine structure of the spermatheca of Apis mellifera. J Insect Physiol 21: 89-109.

den Boer SPA, Boomsma JJ, Baer B 2009. Honey bee males and queens use glandular secretions to enhance sperm viability before and after storage. J Insect Physiol 55: 538-543.

Foster WA, Walker ED 2009. Mosquitoes (Culicidae). In GR Mullen, LA Durden, Medical and veterinary entomology, Elsevier, Amsterdam, p. 207-259.

Fritz AH, Turner FR 2002. A light and electron microscopical study of the spermathecae and ventral receptacle of Anastrepha suspensa (Diptera: Tephritidae) and implications in female influence of sperm storage. Arthropod Struct Dev 30: 293-313.

Gerber HS, Klostermeyer EC 1970. Sex control by bees: a voluntary act of egg fertilization during oviposition. Science 167: 82-84.

Gillot C 2003. Male accessory gland secretions: modulators of female reproductive physiology and behavior. Annu Rev Entomol 48: 163-184.

Giuffrida A, Focarelli R, Lampariello R, Thole H, Rosati F 1995. Purification and properties of a $35 \mathrm{kDa}$ glycoprotein from spermathecal extract of Eyprepocnemis plorans (Insecta: Orthoptera) with axonemal cytoskeleton disassembly activity. Insect Biochem Mol Biol 26: 347-354.

Gobin B, Fumionori I, Peeters C, Billen J 2006. Queen-worker differences in spermatheca reservoir of phylogenetically basal ants. Cell Tissue Res 326: 169-178.

Ilango K 2005. Structure and function of the spermathecal complex in the phlebotomine sandfly Phlebotomus papatasi Scopoli (Diptera: Psychodidae): II. Post-copulatory histophysiological changes during the gonotrophic cycle. J Biosc 30: 711-731.

Jones JC, Wheeler RE 1965. Studies on spermathecal filling in Aedes aegypti (Linnaeus). I. Description. Biol Bull 129: 134-150.

Klenk M, Koeniger G, Koeniger N, Fasold H 2004. Proteins in spermathecal gland secretion and spermathecal fluid and the properties of a $29 \mathrm{kDa}$ protein in queens of Apis mellifera. Apidologie 35: 371-381.

Klowden MJ, Chambers GM 2004. Production of polymorphic sperm by anopheline mosquitoes and their fate within the female genital tract. J Insect Physiol 50: 1163-1170.

Kumano N, Haraguchi D, Kohama T 2009. Sperm storage and viability within females of Euscepes postfasciatus: effect of irradiation on sperm abundance and viability within female. J Insect Physiol 55: 813-817.

Lawson FA, Thomas JC 1970. Spermathecae in Periplaneta americana (Blattaria: Blattidae). J Kansas Entomol Soc 43: 418-434.
Lay M, Zissler D, Hartman ED 1999. Ultrastructural and functional aspects of the spermatheca of the African migratory locust $\mathrm{Lo}$ custa migratoria migratorioides (Reiche and Fairmaire) (Orthoptera: Acrididae). J Insect Morphol Embryol 28: 349-361.

Longin A, Souchier C, Ffrench M, Bryon PA 1993. Comparison of anti-fading agents used in fluorescence microscopy: image analysis and laser confocal microscopy study. J Histochem Cytochem 41: $1833-1840$.

Martins GF, Serrão JE 2002. A comparative study of the spermatheca in bees (Hymenoptera: Apoidea). Sociobiology 40: 711-720.

Martins GF, Serrão JE, Furieri KS 2005. Notes on the spermatheca of Vespidae and Sphecidae (Hymenoptera). Sociobiology 45: 119-127.

Martins GF, Zanúncio ZC, Serrão JE 2008. Spermatheca morphology of the social wasp Polistes erythrocephalus. Bull Insectol 61: 37-41.

Muir LE, Kay BH 1998. Aedes aegypti survival and dispersal estimated by mark-release-recapture in northern Australia. Am J Med Hyg 58: 277-282.

Prokupek A, Hoffmann F, Eyun S, Moriyama E, Zhhou M, Marshman L 2008. An evolutionary expressed sequence tag analysis of Drosophila spermatheca genes. Evolution 62: 2936-2947.

Rogers DW, Whitten MMA, Thailayil J, Soichot J, Levashina EA, Catteruccia F 2008. Molecular and cellular components of the mating machinery in Anopheles gambiae females. Proc Natl Acad Sci USA 105: 19390-19395.

Schoeters E, Billen J 2000. The importance of the spermathecal duct in bumble bees. $J$ Insect Physiol 46: 1303-1312.

Sirot LK, Hardstone MC, Helinski MEH, Ribeiro JMC, Kimura M, Deewatthanawong P, Wolfner MF, Harrington LC 2011. Towards a semen proteome of the dengue vector mosquito: protein identification and potential functions. PLoS Negl Trop Dis 5: e989.

Sirot LK, Poulson RL, McKenna MC, Girnary MFW, Harrington LC 2008. Identity and transfer of male reproductive gland proteins of the dengue vector mosquito, Aedes aegypti: potential tools for control of female feeding and reproduction. Insect Biochem Mol Biol 38: 176-189.

Souza EA, Campos LAO, Neves CA, Zanuncio JC, Serrão JE 2007. Effect of delayed mating on spermathecal activation in Melipona quadrifasciata anthidioides (Hymenoptera: Apidae). Apidologie 39: 293-301.

Staccomi MVR, Romani R 2010. Ultrastructural and functional aspects of the spermatheca in the American harlequin bug, Murgantia histrionica (Hemiptera: Pentatomidae). Neotrop Entomol 40: 222-230.

Strickman D 2006. Longevity of Aedes aegypti (Diptera: Culicidae) compared in cages and field under ambient conditions in rural Thailand. Southeast Asian J Trop Med Publ Health 37: 456-462.

Tejerina EF, Almeida FFL, Almirón WR 2009. Bionomics of Aedes aegypti subpopulations (Diptera: Culicidae) from Misiones Province, northeastern Argentina. Acta Trop 109: 45-49.

Thailayil J, Magnusson K, Godfray HCJ, Crisanti A, Catteruccia F 2011. Spermless males elicit large-scale female responses to mating in the malaria mosquito Anopheles gambiae. Proc Natl Acad Sci USA 108: 13677-13681.

Tombes AS, Roppel RM 1972. Ultrastructure of the spermatheca of the granary weevil, Sitophilus granarius (L.) (Coleoptera: Curculionidae). Int J Insect Morphol Embryol 1: 141-152.

Werner M, Zissler D, Peschke K 1999. Structure and energy pathways of spermatozoa of the rove beetle Aleochara bilineata (Coleoptera, Staphylinida). Tissue Cell 31: 413-420.

Wheeler D, Krutzsch P 1994. Ultrastructure of the spermatheca and its associated gland in the ant Crematogaster opuntiae (Hymenoptera: Formicidae). Zoomorphology 114: 203-212. 